\title{
Indicadores biofísicos de degradação ambiental no Parque Nacional de Sete Cidades, Nordeste do Brasil
}

\author{
Biophysical indicators of environmental degradation in the Parque Nacional de Sete \\ Cidades, Northeast Brazil
}

SANTOS $^{1}$, F.A.; PACIÊNCIA ${ }^{2}$, L.G.M.; MENDES $^{3}$, J.M.; AQUINO ${ }^{4}$, C.M.S. francilio.amorim@ifpi.edu.br

\begin{abstract}
Resumo
O presente estudo propôs-se a analisar o estado de degradação do Parque Nacional (PARNA) de Sete Cidades, através da aplicação de indicadores biofísicos de degradação ambiental, considerando os aspectos geológicos, geomorfológicos, climáticos, pedológicos e fitogeográficos. A referida Unidade de Conservação (UC) situa-se entre os municípios de Piracuruca e Brasileira, Nordeste do estado do Piauí. Para realizar o presente estudo foram adquiridos os seguintes arquivos matriciais e vetoriais: Mapa de geodiversidade do Piauí; imagens da Missão SRTM e dos satélites Landsat 5 TM e 8 OLI; dados de quatro postos pluviométricos para os anos de 1998, 2006 e 2014; e Mapa de solos da Folha SB.24. Tais arquivos foram manuseados através do Sistema de Informações Geográficas (SIG) Quantum GIS (QGIS) versão 2.14 - Essen. Quando integrados os indicadores de degradação - Geologia, Geomorfologia, Clima, Solos e Cobertura Vegetal - foi possível identificar que na Unidade de Conservação estudada há preponderância do índice biofísico de degradação moderado para os anos de 1998, 2006 e 2014. Tal fato deve-se a preponderância de alto a muito alto potencial de Erosividade das chuvas (R) e Erodibilidade dos solos (K) aliados a baixa proteção da cobertura vegetal.
\end{abstract}

Palavras-chave: Unidade de Conservação. Aspectos geoambientais. Índice Biofísico.

\begin{abstract}
This study aimed to analyze the degradation state of the Parque Nacional (PARNA) de Sete Cidades, through the application of biophysical indicators of environmental degradation, considering the geological aspects, geomorphological, climatic, soil and phytogeographic. Said Conservation Unit (CU) is located between the municipalities of Piracuruca and Brasileira, the state of Piauí in the Northeast. To perform this study were purchased the following raster files and vector: Piauí geodiversity map; Images Mission SRTM and satellites Landsat 5 TM and 8 OLI; data from four rain gauges for the years 1998, 2006 and 2014; and SB.24 Leaf Soil Map. These files were handled by Geographic Information System (GIS) Quantum GIS (QGIS) Version 2.14 - Essen. When integrated degradation indicators - Geology, Geomorphology, Climate, Soils and Vegetation Cover - was identified that the Conservation Unit studied for preponderance of biophysical index moderate deterioration for the years 1998, 2006 and 2014. This is due, the preponderance of high to very high potential Erosivity of Rainfall (R) and Soil Erodibility (K) combined with low protection of vegetation cover.
\end{abstract}

Keywords: Conservation Unit. Geo-environmental Aspects. Biophysical Index.

\section{INTRODUÇÃO}

Atualmente, os estudos ambientais têm se utilizado de indicadores biofísicos para análise da paisagem e os mesmos vem ganhando destaque na região do Nordeste, como comprovam os estudos de Lopes e Candeias (2010) e Bezerra et al. (2014).

Lopes e Candeias (2010) utilizaram algoritmos na Linguagem LEGAL (Spring 4.0) para analisar de modo integrado os seguintes indicadores: algoritmos do Albedo, Temperatura, Emissividade, NDVI, WDVI, SAVI, MSAVI. Albedo, NDVI, SAVI e Emissividade. Cujo objetivo foi quantificar 
os processos de degradação na Bacia do Rio Brígida, visando o diagnóstico e distribuição do processo através de aplicação dos parâmetros em imagens Landsat TM.

Bezerra et al. (2014) avaliaram a dinâmica espaço-temporal por meio de técnicas de sensoriamento remoto do Parque Nacional da Furna Feia, entre Mossoró e Baraúna, RN. Utilizaram-se de imagens orbitais do satélite Landsat 5 TM dos anos de 2007 e 2010. Concluíram que a variação de albedo, da temperatura da superfície e do NDVI seguem o padrão esperado. Identificaram diferenças nos padrões espaciais e temporais dos parâmetros biofísicos e alto poder de resiliência, devido à grande produção de biomassa no inverno.

Os trabalhos apresentados apontam a importância do uso de indicadores biofísicos como forma de quantificar, avaliar e monitorar os processos de degradação ambiental, em especial no semiárido. Neste interim o presente estudo buscou analisar o estado de degradação do Parque Nacional (PARNA) de Sete Cidades, localizado no Nordeste do estado do Piauí, através da aplicação de indicadores biofísicos de degradação ambiental, considerando a Litologia, Declividade, Erosividade das chuvas, Erodibilidade dos solos e Índice de Vegetação por Diferença Normalizada.

\section{METODOLOGIA}

\subsection{Localização e caracterização da área em estudo}

O PARNA de Sete Cidades situa-se no Nordeste do estado do Piauí, entre os municípios de Brasileira e Piracuruca, especificamente às Coordenadas Geográficas $04^{\circ} 05^{\prime} \mathrm{S}$ e $04^{\circ} 15^{\prime} \mathrm{S}$ e $41^{\circ} 30^{\prime} \mathrm{W}$ e $41^{\circ} 45^{\prime} \mathrm{W}$, conforme exposto na Figura 01. A referida Unidade de Conservação (UC) possui 6.221 hectares de extensão e perímetro que compreende 36,2 km (BRASIL, 1979).

O Parque Nacional de Sete Cidades apresenta afloramentos rochosos maciços ou ruiniforme, lajeado, pavimentação de blocos, formações arenosas e couraça ferruginosa e solos hidromórficos (SANTOS e PELLERIN, 2006). Suas precipitações pluviométricas médias anuais são estimadas em torno de $1.500 \mathrm{~mm}$ (BRASIL, 1979), pois estas sofrem influência da Zona de Convergência Intertropical (ZCIT). As ordens de solos identificados foram as seguintes: Plintossolo Pétrico Concrecionário, Neossolo Litólico Distrófico e Neossolo Quartzarênico Órtico (INDE, 2014). Estes solos são recobertos por: i) vegetação do tipo floresta tropical ombrófila aluvial ocasionalmente inundada, ii) floresta tropical semidecídua, iii) floresta aberta latifoliada perenifólia, iv) cerrado aberto latifoliado perenifólio, v) cerrado extremamente xeromórfico e vi) campo graminóide cespitoso médio (OLIVEIRA et. al. 2007). Tais elementos quando integrados resultam numa paisagem modelada pelos elementos climáticos, em especial as precipitações, que modelam o relevo e condicionam a fisionomia da cobertura vegetal. 


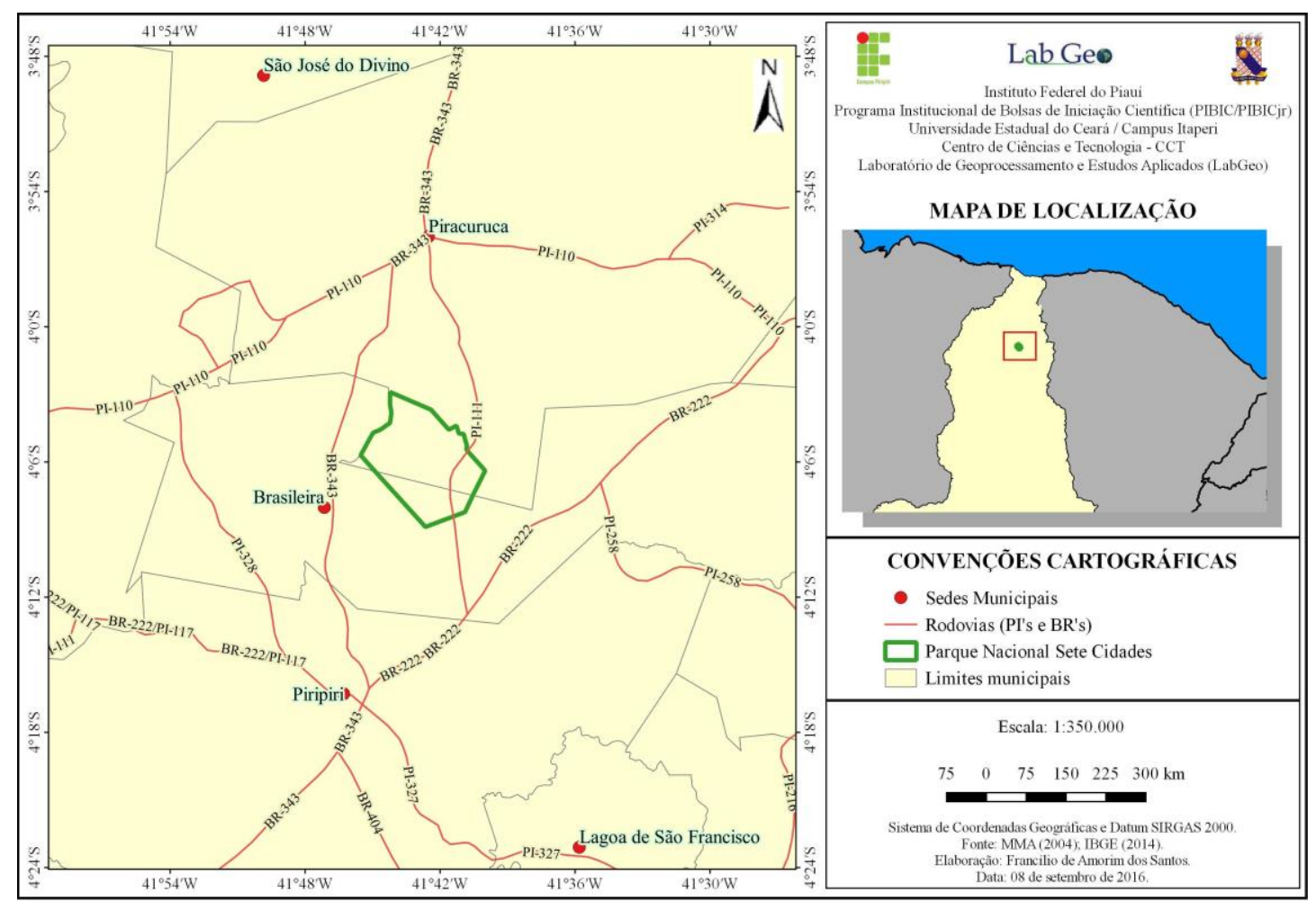

Figura 01. Localização do Parque Nacional de Sete Cidades, obtido a partir dos dados geográficos do MMA (2004) e da malha municipal digital do Brasil, em escala 1:250.000 do IBGE (2014).

\subsection{Procedimentos metodológicos}

A degradação ambiental do PARNA de Sete Cidades foi avaliada mediante a aquisição de arquivos matriciais e vetoriais, pois são as informações disponibilizadas pelos órgãos governamentais. Desse modo, tais os dados sofrem adaptações em relação à proposta metodológica original de Oliveira (2011), devido principalmente à ausência de dados em escala mais adequada ao tamanho da área em estudo.

Os procedimentos para identificar as áreas absolutas e relativas para cada indicador biofísico, conforme é exposto no Quadro 01, e composição do índice biofísico de degradação ambiental foram realizados através das ferramentas do Sistema de Informação Geográfica (SIG) Quantum GIS $(Q G I S)$, versão 2.14 - Essen. Desse modo, foram utilizados os seguintes dados:

Mapa de geodiversidade do Piauí da CPRM (2006), em esca1a: 1.000.000, disponível em http://geobank.sa.cprm.gov.br, utilizado para mensuração do parâmetro geológico;

$\checkmark$ Modelo Digital de Elevação (MDE) da Shuttle Radar Topographic Mission (SRTM), com resolução espacial de 30 metros, obtido no site do United States Geological Service (USGS), disponível em http://earthexplorer.usgs.gov/; para avaliação do parâmetro geomorfológico; 
Quadro 01. Indicadores biofísicos propostos para avaliação da degradação ambiental do Parque Nacional de Sete Cidades. Fonte: Oliveira (2011). ${ }^{1}$ Conforme proposto em Ross (1994); ${ }^{2}$ De acordo com Santos (2015); ${ }^{3}$ Segundo a classificação proposta por Crepani et al. (2001); ${ }^{4}$ Consoante ao proposto por Melo et al. (2011).

\begin{tabular}{|c|c|c|}
\hline Geologia (Litotipos) - IBFD1 & Faixa & V.I \\
\hline Arenitos & - & (4) \\
\hline Geomorfologia (Declividade ${ }^{1}$ ) - IBFD2 & Faixa & V.I \\
\hline Muito Fraca & $<6 \%$ & $(5)$ \\
\hline Fraca & 6 a $12 \%$ & (4) \\
\hline Média & 12 a $20 \%$ & (3) \\
\hline Forte & 20 a $30 \%$ & (2) \\
\hline Muito Forte & $>30 \%$ & (1) \\
\hline Clima (Fator $\mathbf{R}-$ Erosividade $^{2}$ ) - IBFD3 & Faixa & V.I. \\
\hline Média & $8.162,0$ a $8.297,9$ MJ.mm/ha.h.ano & (3) \\
\hline Alta & 8.297,9 a $8.433,6$ MJ.mm/ha.h.ano & (2) \\
\hline Muito Alta & $8.433,6$ a $8.569,2$ MJ.mm/ha.h.ano & (1) \\
\hline Solos $\left(\right.$ Fator K - Erodibilidade ${ }^{3}$ ) - IBFD4 & Faixa & V.I. \\
\hline Alta & - & $(2)$ \\
\hline Muito Alta & - & (1) \\
\hline Cobertura Vegetal $\left(\right.$ NDVI $\left.^{4}\right)$ - IBFD5 & Faixa & V.I \\
\hline 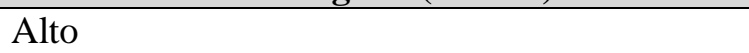 & $0,6 a<0,8$ & (4) \\
\hline Médio & $0,4 a<0,6$ & (3) \\
\hline Baixo & $0,2 \mathrm{a}<0,4$ & (2) \\
\hline Muito Baixo & $>0 a<0,2$ & (1) \\
\hline
\end{tabular}

Dados de quatro postos pluviométricos da Agência Nacional de Águas (ANA), considerando a série histórica de 1998 a 2014, disponível em http://hidroweb.ana.gov.br/; esses dados foram utilizados para conhecimento da Erosividade das chuvas (R);

$\checkmark$ Mapa de solos da Folha SB.24 da Infraestrutura de Dados Espaciais (INDE, 2014), disponível em http://www.visualizador.inde.gov.br/; utilizado para avaliação da Erodibilidade dos solos;

$\checkmark$ Imagens via satélites Landsat 5 TM e Landsat 8 OLI, com resolução espacial de 30 metros, disponível no site http://earthexplorer.usgs.gov/ do USGS, com órbita/ponto 219/63 e as datas de passagem: 23 de agosto de 1998, 29 de agosto de 2006 e 19 de agosto de 2014.

\subsubsection{IBFD1 - Indicador Biofísico Geologia (Litotipos)}

Através desse indicador é possível perceber a resistência das rochas aos processos de intemperismos. A identificação da litologia em Sete Cidades baseou-se no Mapa de geodiversidade do Piauí (CPRM, 2006) e sua classificação deu-se de acordo com a proposta de Oliveira (2011).

\subsubsection{IBFD2 - Indicador Biofísico Geomorfologia (Declividade Média - DM)}

De acordo com Ross (1994), a análise da fragilidade é composta por um conjunto de estudos/produtos intermediários (inclusos nesse trabalho e também em outros indicadores). Nesse trabalho a geomorfologia será representada pela declividade e identificada com base o MDE SRTM. 


\subsubsection{IBFD3 - Indicador Biofísico Clima (Erosividade das Chuvas - R)}

Nesse estudo a variável climática baseou-se na Erosividade das chuvas (R), essa se baseia em técnica de regressão linear entre o índice médio mensal de erosão e o coeficiente de chuva, estimada mediante aplicação da Equação 1 (LOMBARDI NETO e MOLDENHAUER, 1992).

$$
\mathrm{E}=67,355\left(\mathrm{r}^{2} / \mathrm{P}\right)^{0,85}
$$

Onde: $\mathrm{E}=$ média mensal do índice de erosão (t/ha.mm/h); $\mathrm{r}=$ precipitação média mensal em $\mathrm{mm} ; \mathrm{P}=$ precipitação média anual em $\mathrm{mm}$.

Para análise do fator R adquiriu-se dados de precipitação média anual das séries anuais (1998 a 2014) de quatro postos pluviométricos da ANA (2016), conforme exposto no Quadro 02. As falhas das séries foram corrigidas através da técnica de Ponderação Regional proposto por Tucci (1993) e uso do pacote de programas USUAIS (OLIVEIRA e SALES, 2016).

Quadro 02. Postos pluviométricos utilizados para espacialização da Erosividade das chuvas (R) no Parque Nacional de Sete Cidades. Fonte: ANA (2016).

\begin{tabular}{|c|c|c|c|c|}
\hline Nome do posto & Fonte & Município & Latitude & Longitude \\
\hline Batalha & DNOCS & Batalha & $-04^{\circ} 20^{\prime} 0.00^{\prime \prime} \mathrm{W}$ & $-42^{\circ} 05^{\prime} 0.00^{\prime \prime} \mathrm{S}$ \\
\hline Piracuruca & DNOCS & Piracuruca & $-03^{\circ} 56^{\prime} 0.00^{\prime \prime} \mathrm{W}$ & $-41^{\circ} 43^{\prime} 0.00^{\prime \prime} \mathrm{S}$ \\
\hline Piripiri & DNOCS & Piripiri & $-04^{\circ} 17^{\prime} 0.00^{\prime \prime} \mathrm{W}$ & $-41^{\circ} 47^{\prime} 0.00^{\prime \prime} \mathrm{S}$ \\
\hline Tinguis & CPRM & Piracuruca & $-03^{\circ} 43^{\prime} 2.40^{\prime \prime} \mathrm{W}$ & $-41^{\circ} 58^{\prime} 3.20^{\prime \prime} \mathrm{S}$ \\
\hline
\end{tabular}

\subsubsection{IBFD4 - Indicador Biofísico Solos (Erodibilidade dos solos - K)}

$\mathrm{O}$ fator $\mathrm{K}$ que corresponde à Erodibilidade dos solos foi utilizado para identificar a suscetibilidade dos solos frente ao desprendimento e transporte pelos agentes de erosão. $\mathrm{O}$ arquivo utilizado para tal fim foi o Mapa de Solos da Folha SB.24 - Jaguaribe, ao passo que as classes estabelecidas para o fator K consideraram a metodologia proposta em Crepani et al. (2001).

\subsubsection{IBFD5 - Indicador Biofísico Cobertura Vegetal (Índice de Vegetação por Diferença} Normalizada - NDVI)

A determinação do índice de cobertura vegetal é extremamente importante, visto que a vegetação tem relação direta com a redução da energia cinética das gotas de águas das chuvas. No presente estudo considerou-se o Índice de Vegetação por Diferença Normalizada (NDVI) para análise do nível de proteção da cobertura vegetal do PARNA em questão. O NDVI constou da aplicação da Equação 2 descrita por Jensen (1996, apud MELO et al., 2011): 


$$
\mathrm{NDVI}=(\mathrm{NIR}-\mathrm{R}) /(\mathrm{NIR}+\mathrm{R})
$$

Em que: NDVI = Índice de Vegetação por Diferença Normalizada; NIR = refletância no comprimento de onda correspondente ao Infravermelho Próximo $(0,76$ a $0,90 \mu \mathrm{m}$ para o Landsat 5 e $0,85$ a $0,88 \mu \mathrm{m}$ para o Landsat 8$) ; \mathrm{R}=$ refletância no comprimento de onda correspondente ao Vermelho (0,63 a 0,69 $\mu \mathrm{m}$ para o Landsat 5 e 0,64 a 0,67 $\mu \mathrm{m}$ para o Landsat 8).

Nesse estudo, foram selecionadas três cenas que correspondem aos anos de 1998, 2006 e 2014. Destaca-se que as imagens que foram adquiridas junto ao USGS, possuem resolução espacial de 30 metros. Considerou-se, ainda, a menor presença de nuvens para a escolha das referidas cenas, essas possuem órbita/ponto 219/63 e as seguintes datas de passagem: 23 de agosto de 1998, 29 de agosto de 2006 e 19 de agosto de 2014. Destaca-se que as imagens passaram por processos de conversão geométrica e radiométrica (16 para 8 bits) e reclassificadas através das ferramentas do QGIS, permitindo melhorar a representação do índice de vegetação.

\section{RESULTADOS E DISCUSSÃO}

\subsection{Análise dos indicadores biofísicos de degradação ambiental}

Inicialmente, pode-se afirmar que no PARNA há preponderância de litologia arenítica, em 100\% da área (BRASIL, 1979). Propiciando-se, de acordo com Penteado (1980), uma maior resistência ao escoamento superficial e, também, aos processos naturais de intemperismo.

Os dados de declividade do relevo apontaram predomínio das categorias muito baixa a baixa em 83,9\% no PARNA estudado (Tabela 01). Destaca-se, ainda, que foi encontrado relevo com declividade alta a muito alta em apenas 6,8\% da Unidade de Conservação, representada principalmente pelos morros e colinas que ocorrem na área. Nesse sentido, pode-se afirmar que a maior parte do relevo apresenta baixo potencial a processos de escorregamento e/ou deslizamento.

Tabela 01. Categorias hierárquicas e pesos da declividade do relevo do Parque Nacional de Sete Cidades.

\begin{tabular}{c|c|c|c}
\hline Classes de fragilidade do relevo & Peso & Área (ha) & \% \\
\hline Muito Baixa & 1 & $4.105,9$ & 66,0 \\
\hline Baixa & 2 & $1.113,6$ & 17,9 \\
\hline Média & 3 & 578,5 & 9,3 \\
\hline Alta & 4 & 311,0 & 5,0 \\
\hline Muito Alta & 5 & 112,0 & 1,8 \\
\hline \multicolumn{2}{|c|}{ Total } & $\mathbf{6 . 2 2 1}$ & $\mathbf{1 0 0}$ \\
\hline
\end{tabular}

No que tange ao fator R, considerando a série histórica de 1998 a 2104, pode-se afirmar que em Sete Cidades predominam as classes alta a muito alta Erosividade das chuvas, que se distribui por 
4.304,9 $\mathrm{km}^{2}(69,2 \%)$ da área. Ao passo que a classe de média Erosividade é encontrada em 1.916,1 $\mathrm{km}^{2}(30,8 \%)$ da referida UC. Os altos valores encontrados para o fator $\mathrm{R}$ devem-se aos significativos totais de precipitação pluviométricas concentradas em poucos meses do ano.

Por sua vez, o componente pedológico do PARNA indicou predomínio de alto a muito alto potencial de Erodibilidade de seus solos. Esse alto a muito alto potencial justifica-se pela presença de solos jovens e pouco desenvolvidos, quais sejam: Neossolo Quartzarênico Órtico, Plintossolo Pétrico Concrecionário e Neossolo Litólico Distrófico que recobrem, respectivamente, 75,5\%, 14,6\% e 9,9\% da Unidade de Conservação (INDE, 2015).

A partir da Tabela 02, pode-se notar que dentre os valores de NDVI há predomínio da classe de baixa proteção em 72,2\%, 51,9\% e 85\% da área, respectivamente, para os anos de 1998, 2006 e 2014. Entretanto, a classe baixa teve uma queda de 20\% do ano 1998 para 2006. Destaca-se que a redução das classes de muito baixa e baixa proteção dos anos de 1998 e 2006, implicou em aumento da classe de proteção média, pois esta passou de 14,8\% em 1998 para 44,8\% em 2006. Ressalta-se que entre os anos de 2006 e 2014 houve uma diminuição em 44,6\% da classe média, ao passo que a classe de baixa e muito baixa proteção ocorreram, respectivamente, em 85\% e 14,8\% da área. Os dados de NDVI relativos a 2014 evidenciam um aumento do risco de degradação, posto ter havido redução da biomassa da cobertura vegetal.

Tabela 02. Classes de fragilidade e pesos da cobertura vegetal (NDVI) no Parque Nacional de Sete Cidades, comparação para anos de 1998, 2006 e 2014. Fonte: Organizados pelos autores (2016).

\begin{tabular}{c|c|c|c|c|c|c|c}
\hline $\begin{array}{c}\text { Classes da Cobertura } \\
\text { Vegetal (NDVI) }\end{array}$ & \multirow{2}{*}{ Peso } & \multicolumn{2}{|c|}{$\mathbf{1 9 9 8}$} & \multicolumn{2}{c|}{$\mathbf{2 0 0 6}$} & \multicolumn{2}{c}{$\mathbf{2 0 1 4}$} \\
\cline { 3 - 8 } & & Área (ha) & $\mathbf{\%}$ & Área (ha) & $\mathbf{\%}$ & Área (ha) & \% \\
\hline Alta & 1 & 43,5 & 0,7 & 12,4 & 0,2 & - & - \\
\hline Média & 2 & 920,7 & 14,8 & 2787,0 & 44,8 & 12,5 & 0,2 \\
\hline Baixa & 3 & $4.491,6$ & 72,2 & 3228,7 & 51,9 & 5287,8 & 85,0 \\
\hline Muito Baixa & 4 & 765,2 & 12,3 & 192,9 & 3,1 & 920,7 & 14,8 \\
\hline \multicolumn{2}{|c|}{ Total } & $\mathbf{6 . 2 2 1}$ & $\mathbf{1 0 0}$ & $\mathbf{6 . 2 2 1}$ & $\mathbf{1 0 0}$ & $\mathbf{6 . 2 2 1}$ & $\mathbf{1 0 0}$ \\
\hline
\end{tabular}

Deve-se destacar que as variações nas classes de NDVI podem está associadas às oscilações dos volumes pluviométricos médios anuais, considerando os anos de 1998, 2006 e 2014, conforme dados pluviométricos da ANA (2016). Logo, interferindo diretamente no porte e biomassa apresentada pela vegetação, logo deixando os solos mais desprotegidos. Pois a média pluviométrica anual registrada para 1998 foi 1.222,6 mm anuais, 1.442,8 mm em 2006 e 1.270,9 mm em 2014.

\subsection{Análise do índice biofísico degradação ambiental}

Considerando adaptações à metodologia original proposta por Oliveira (2011), acerca dos indicadores biofísicos de degradação, foi possível compor o índice biofísico de degradação (Quadro 
03). Destaca-se que devido à predominância da classe de baixa proteção da cobertura vegetal o PARNA estudado apresentou o mesmo índice biofísico de degradação para 1998, 2006 e 2014.

Quadro 03. Indicadores biofísicos de degradação ambiental do Parque Nacional de Sete Cidades. Fonte: Oliveira (2011). Adaptado pelos autores (2016).

\begin{tabular}{|c|c|c|c|c|c|c|}
\hline \multirow{2}{*}{ Anos } & \multicolumn{7}{|c|}{ Indicadores biofísicos de degradação ambiental } \\
\cline { 2 - 7 } & IBFD1 & IBFD2 & IBFD3 & IBFD4 & IBFD5 & IBFD \\
\hline $\mathbf{1 9 9 8 , 2 0 0 6}$ e 2014 & 5 & 4 & 1 & 2 & 3 & 3,0 \\
\hline
\end{tabular}

Diante do exposto, foi possível apontar que o PARNA de Sete Cidades possui um índice biofísico de degradação considerado moderado. Pois quando integrado os indicadores biofísicos os valores dos pesos situaram-se no intervalo de 2,80 a 3,19. Deve-se destacar que o IBFD3 (Erodibilidade), IBFD4 (Erosividade) e IBFD5 (NDVI) foram os que contribuíram com maior peso para o índice biofísico, pois apresentaram alto a muito alto potencial dos fatores $\mathrm{R}$ e $\mathrm{K}$ associados a uma baixa proteção da cobertura vegetal. Por outro lado, os IBFD1 (Litologia) e IBFD2 (Declividade) contribuíram para atenuar os efeitos dos outros indicadores, principalmente, devido à preponderância de litologia arenítica, que oferecem maior resistência, e relevo plano, que constitui baixo potencial a escorregamentos e/ou deslizamentos.

\section{CONSIDERAÇÕES FINAIS}

Os resultados encontrados permitiram conhecer o índice de degradação no Parque Nacional de Sete Cidades e, ainda, reconhecer a importância de estudos ambientais baseados no uso de indicadores biofísicos para avaliação da degradação ambiental em Unidades de Conservação. Nesse contexto, foi possível identificar que na área em estudo predomina: litologia arenítica em 100\% da área; relevo com declividades muito baixa a baixa, distribuídas por $83,9 \%$; fatores $\mathrm{R}$ e $\mathrm{K}$ com alto a muito alto potencial, respectivamente, em 69,2\% e 100\% de sua área, o primeiro devido à concentração dos totais pluviométricos em curto período de tempo e o segundo à presença de solos jovens e pouco desenvolvidos - Neossolo Quartzarênico Órtico, Plintossolo Pétrico Concrecionário e Neossolo Litólico Distrófico; classe de baixa proteção da cobertura vegetal, havendo apenas variações entre os anos considerados, pois a mesma distribuía-se por 72,2\%, 51,9\% e 85\% da área, respectivamente, em 1998, 2006 e 2014.

A integração dos indicadores de degradação permitiu inferir que em Sete Cidades predomina o índice biofísico de degradação considerado moderado para 1998, 2006 e 2014, cuja escala temporal considerou apenas a cobertura vegetal. Ressalta-se que os IBFD3, IBFD4 e IBFD5 contribuíram com maior peso para o índice biofísico, devido predomínio de alto a muito alto potencial dos fatores 
$\mathrm{R}$ e K que aliados a uma baixa proteção da cobertura vegetal acentuam o potencial de degradação na Unidade de Conservação. Entretanto, os IBFD1 e IBFD2 reduziram os efeitos dos outros indicadores, devido à presença de litologia arenítica, com maior resistência ao escoamento superficial, e relevo plano com baixo potencial a processos de escorregamento e/ou deslizamento.

\section{REFERÊNCIAS}

ANA - Agência Nacional de Águas. Hidro Web - Sistema de Informações Hidrológicas. Séries históricas - ano de 1998, 2006 e 2014. Disponível em <http://hidroweb.ana.gov.br/>. Acesso em 25 de junho de 2016.

BEZERRA, J. M.; MOURA, G. B. de A.; SILVA, B. B. da; LOPES, P. M. O.; SILVA, E. F. de F. Parâmetros biofísicos obtidos por sensoriamento remoto em região semiárida do estado do Rio Grande do Norte, Brasil. Revista Brasileira de Engenharia Agrícola e Ambiental. Campina Grande, PB, v.18, n.1, p. 73-84, 2014.

BRASIL. Ministério da Agricultura. IBDF - Instituto Brasileiro de Desenvolvimento Florestal. Fundação Brasileira para a Conservação da Natureza. Plano de Manejo do Parque Nacional de Sete Cidades. Doc. Téc. No 1. Brasília. 1979.

CPRM - Companhia de Pesquisa de Recursos Minerais. Mapas estaduais de geodiversidade: Piauí. Rio de Janeiro: CPRM. 2006. Documento cartográfico em arquivo vetorial. Disponível em <http://geobank.sa.cprm.gov.br〉. Acesso em janeiro de 2014.

CREPANI, E.; MEDEIROS, J.S.; HERNANDEZ FILHO, P.; FLORENZANO, T.G.;DUARTE, V.; BARBOSA, C.C.F. Sensoriamento Remoto e Geoprocessamento Aplicados ao Zoneamento Ecológico-Econômico e ao Ordenamento Territorial. São José dos Campos: INPE, 2001.

IBGE - Instituto Brasileiro de Geografia e Estatística. Malha municipal digital do Brasil: situação em 2014. Rio de Janeiro: IBGE, 2014. Disponível em: 〈ftp://geoftp.ibge.gov.br/malhas_digitais/>. Acesso em 04 de abril de 2016.

INDE - Infraestrutura Nacional de Dados Especiais. Mapa de Solos da Folha SB.24 - Jaguaribe. Escala 1:250.000. Disponível em <http://www.visualizador.inde.gov.br/>. 2014. Acesso em 27 de novembro de 2015.

LOMBARDI NETO, F.; MOLDENHAUER, W.C. Erosividade da chuva: sua distribuição e relação com perdas de solos em Campinas - SP. Bragantina, Campinas, v. 51, nº 2, p.189-1996, 1992.

LOPES, H.; CANDEIAS, A. L. B. O uso de parâmetros biofísicos na quantificação e identificação de risco a desertificação na bacia do rio brigida. Zoneamento agroecológico do estado de Pernambuco. 2001.

MMA - Ministério do Meio Ambiente dos Recursos Hídricos e da Amazônia Legal. Download de dados geográficos. 2004. Disponível em <http://mapas.mma.gov.br/i3geo/datadownload.htm>. Acesso em Acesso em 27 de agosto de 2015. 
MELO, E.T.; SALES, M.C.L.; OLIVEIRA, J.G.B. Aplicação do índice de Vegetação por Diferença Normalizada (NDVI) para análise da degradação ambiental da microbacia hidrográfica do riacho dos cavalos, Crateús-CE. Revista RA'E GA, v.23, Curitiba, Departamento de Geografia - UFPR. p.520-533. 2011.

OLIVEIRA, J.G.B.; SALES, M.C.L. Usuais: programas para uso em análise ambiental. Revista Equador (UFPI), Vol. 5, n. 2, p.36-60, Janeiro/Junho, 2016.

OLIVEIRA, M.E.A.; MARTINS, F.R.; CASTRO, A.A.J.F.; SANTOS, J.R. Classes de cobertura vegetal do Parque Nacional de Sete Cidades (transição campo-floresta) utilizando imagens TM/Landsat, NE do Brasil. In: Anais XIII Simpósio Brasileiro de Sensoriamento Remoto, Florianópolis, Brasil, INPE. 21-26 abril 2007.

OLIVEIRA, V.P.V. Indicadores biofísicos de desertificação, Cabo Verde/África. Revista Mercator, Fortaleza, v. 10, n. 22, p.147-168, mai./ago. 2011.

PENTEADO, M.M. Fundamentos de geomorfologia. Rio de Janeiro. IBGE, 1980.

ROSS, J.L.S. Análise empírica da fragilidade dos ambientes naturais e antropizados. Revista do Departamento de Geografia. São Paulo: FFLCH/USP, n. 8, p.63-74, 1994.

SANTOS, F.A. Mapeamento das unidades geoambientais e estudo do risco de degradação/desertificação nos municípios de Castelo do Piauí e Juazeiro do Piauí. Dissertação (Mestrado em Geografia) - Programa de Pós-Graduação em Geografia (PPGGEO) / Universidade Federal do Piauí. 2015.

SANTOS, J.; PELLERIN, J. Feições geomorfológicas do Parque Nacional de Sete Cidades, Piauí. In: VI Simpósio Nacional de Geomorfologia, Goiânia - GO, 6 a 10 de setembro de 2006.

TUCCI, C.E.M. Hidrologia: ciência e aplicação. Porto Alegre: Eds. da UFRGS e da USP, 1993.

USGS - United States Geological Service (Serviço Geológico dos Estados Unidos). Earth Explorer - Collection - Landsat Archive. Disponível em 〈http://earthexplorer.usgs.gov/>. Acesso em 27 de novembro de 2015.

Earth Explorer - Digital Elevation - SRTM 1 Arc-Second Global. 2015. Disponível em http://earthexplorer.usgs.gov/. Acesso em 23 de novembro de 2015.

\section{AGRADECIMENTOS}

Agradecemos ao Instituto Federal do Piauí, pelo financiamento da pesquisa via Edital $\mathrm{n}^{\circ}$ 57/2015, referente ao Programa Institucional de Bolsas de Iniciação Científica. Ao Laboratório de Geoprocessamento e Estudos Aplicados, da Universidade Estadual do Ceará / Campus Itaperi, pelo subsídio do espaço físico e conhecimentos para elaboração dos mapas utilizados no presente estudo.

Recebido em: 14/08/2016 
Santos et al, REGNE, Vol. 2, $N^{o}$ Especial (2016)

Aceito para publicação em: 01/10/2016 